\title{
2017 Reviewer List
}

W

e would like to thank the following reviewers, all of whom have completed reviews for the Journal over the past year (September 1, 2016-August 31, 2017). Without your invaluable support and dedication, we would not be the Journal we are today. On behalf of Sports Health, thank you!

\author{
Siraj Abdullah \\ Kelly L. Adler \\ Ayoola Ibifubara Aiyegbusi \\ Andrew W. Albano \\ Sheila M. Algan \\ Louis Almekinders \\ Bara Alsalaheen \\ Steven B. Ambler \\ Annunziato Amendola \\ Allen F. Anderson \\ Kelley Anderson \\ Robert B. Anderson \\ Jack Taylor Andrish \\ Philip A. Anloague \\ Scott Annett \\ Ivan Antosh \\ Brent Arnold \\ Kyle T. Aune \\ Krista G. Austin \\ Tariq Awan \\ Olufemi Ayeni \\ Zafar Azeem \\ Lane Bailey \\ Robert J. Baker \\ Ryan Baker \\ Volga Bayrakci Tunay \\ Jonathan A. Becker \\ Michael Behringer \\ David R. Bell \\ Michael F. Bergeron \\ John A. Bergfeld \\ Krystian Bigosinski \\ Michael E. Biller \\ Tab Blackburn
}

Andrew Blackman
Nicole Boniquit
David Braunreiter
M. Alison Brooks
Robert H. Brophy
Cathleen Brown Crowell
James Brown
Thomas Buckley
Matthew L. Busam
Jeffrey R. Bytomski
E. Lyle Cain
Aaron Campbell
Rebecca Carl
Douglas Casa
Kyle J. Cassas
Peter N. Chalmers
Rachel Chamberlain
Alison Biercevicz Chambers
Jim Chesnutt
Nicole Chimera
Susan A. Chinworth
Yvonne Chow
Jordan S. Chun
James R. Clugston
Rachel A. Coel
Brian J. Cole
Brian Coleman
Nailah Coleman
Sean Colio
Frank A. Cordasco
Michael Coughlin
Alexander Creighton
Jason W. Deck
Tom Decoster

Nicholas Defauw
Scott Dembowski
Natasha Desai
Laura Diamond
Lindsay DiStefano
Dan Divilbiss
Sameer Dixit
Robert Donatelli
Jeanne Doperak
Scott F. Dye
Jennifer Earl-Boehm
Yoshimi Endo
William Etchison
Mathew Failla
Ted A. Farrar
Ken Fechner
Krzysztof Ficek
Dave Fortenbaugh
Peter J. Fowler
Joel Fuller
Marc T. Galloway
Ted J. Ganley
Jim Garrick
Christian Geannette
Mandeep Ghuman
Charles E. Giangarra
Margaret Elizabeth Gibson
Thomas J. Gill
Brian D. Giordano
Kim Gladden
Neal Glaviano
Josh Timothy Goldman
Marci A. Goolsby
Shiho Goto




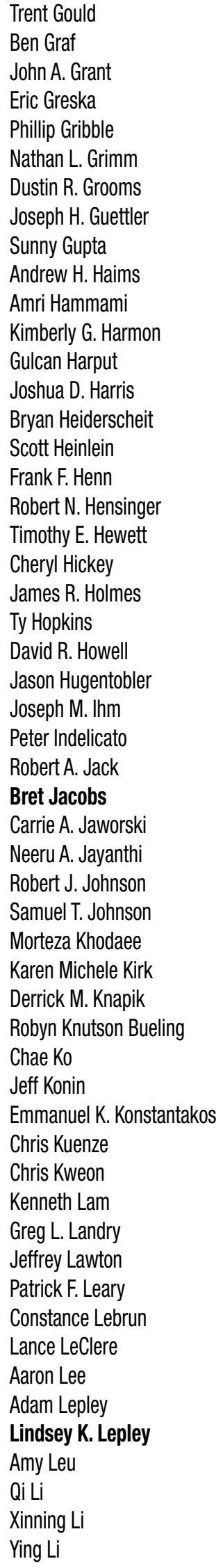

Thomas N. Lindenfeld
Pawel Linek
David K. Lisle
Jeremy P. Loenneke
Robert C. Lynall
C. Benjamin Ma
Bert R. Mandelbaum
Robert C. Manske
Britt Marcussen
Matthew Matava
Neilson M. Mathews
Jason M. Matuszak
David McAllister
Joshua R. McCormack
Patrick C. McCulloch
Timothy McGuine
Chris Miars
Lyle Micheli
Eryn K. Milian
Michael D. Milligan
Jonathan Minor
Monique Mokha
Edward P. Mulligan
Tenley Murphy
Nicholas Murray
Heather Myers
Matthew Mymern
Kyle B. Nagle
Melissa Nayak
Guy Nicolette
Grant Norte
Melissa Novak
Ogonna Kenechi Nwawka
Darell Ogilvie-Harris
Daniel Fulham O'Neill
John Orchard
Kenichi Otoshi
Sakiko Oyama
Patrick Pabian
Ajay Padaki
Dean Padavan
Ronald Paik
Marco Pang
Stephen R. Paul
Bernadette Pendergraph
Ryan Petering
Charles Smith Peterson
Kyle Peterson
Pietro Picerno
Eric Pifel
Marisa Pontillo
Bill Post
Eric Post
David E. Price

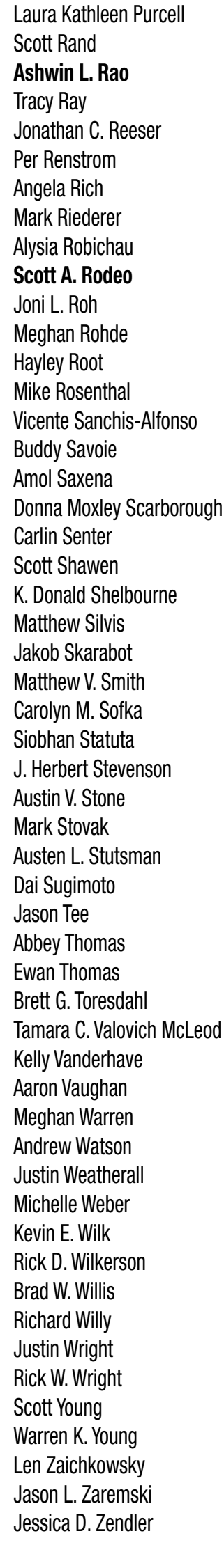

*Bold reviewer names indicate 3 or more reviews completed. 